\title{
Estimation of permeability tensor and dielectric permittivity of ferrites using a wave guide method under a dc magnetic field
}

\author{
I. Tsiachristos ${ }^{1,2}$, E. Varouti ${ }^{2}$, E. Manios ${ }^{2}$, D. Stamopoulos ${ }^{2}$, T. Zervos ${ }^{3}$, G. Fikioris ${ }^{1}$, F. Lazarakis ${ }^{3}$, A. Alexandridis ${ }^{3}$ \\ and M. Pissas ${ }^{2, a}$ \\ ${ }^{1}$ School of Electrical and Computer Engineering, NTUA, Heroon Polytechniou 9, 15780 Zografou, Greece. \\ ${ }^{2}$ IAMPPNM, NCSR “Demokritos”, 15310, Athens, Greece. \\ ${ }^{3}$ IIT, NCSR "Demokritos”, 15310 Athens, Greece
}

\begin{abstract}
Using a vector network analyzer equipped with a calibrated rectangular wave guide the electric permittivity and the element of the magnetic permeability tensor for $\mathrm{Y}_{3} \mathrm{Fe}_{5} \mathrm{O}_{12}, \mathrm{ZnFe}_{2} \mathrm{O}_{4}$ and $\mathrm{NiFe}_{2} \mathrm{O}_{4}$ are measured. The electric permittivity can be estimated from the body resonances $(d=n \lambda / 2)$ if a sufficient long sample is used. The estimation of the magnetic permeability tensors' parameters can be estimated comparing the experimental results with computer simulations using the magnetic properties of the materials as derived from the magnetic measurements.
\end{abstract}

\section{Introduction}

The role of magnetically soft ferrites with Neel temperature larger than $300 \mathrm{~K}$ is very important in microwave devices.

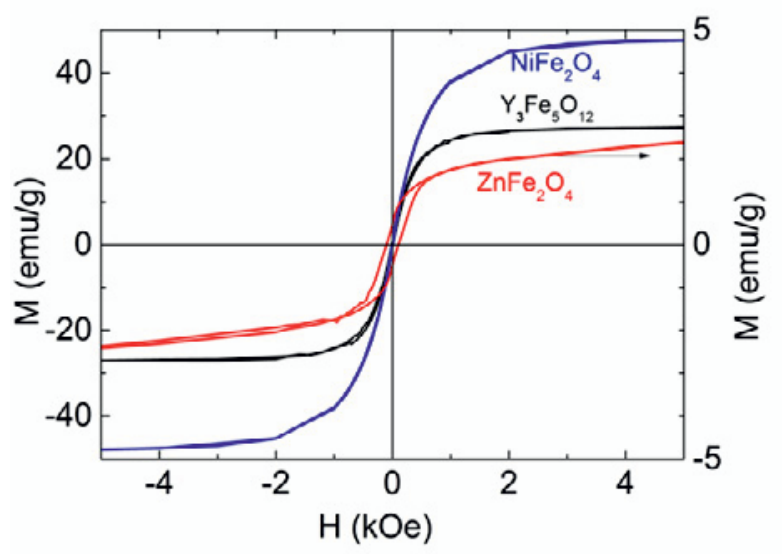

Fig. 1. Magnetic moment per g, versus external magnetic field, at $\mathrm{T}=300 \mathrm{~K}$ for $\mathrm{Y}_{3} \mathrm{Fe}_{5} \mathrm{O}_{12}, \mathrm{NiFe}_{2} \mathrm{O}_{4}$ and $\mathrm{ZnFe}_{2} \mathrm{O}_{4}$. Note the small saturation magnetization of $\mathrm{ZnFe}_{2} \mathrm{O}_{4}$.

Typical examples are circulators, phase shifters and isolators [1,2]. Moreover it has been demonstrated that magnetized ferrites in patch antennas induce novel properties [3]. The presence of the ferrites in the substrate of the antenna can tune the polarization of the radiated

\footnotetext{
${ }^{a}$ Corresponding author: mpissas@ims.demokritos.gr
}

field as well as other characteristics of the antenna in a controllable way through an external magnetic field. To facilitate the design of novel devices knowledge of electrical permittivity and magnetic permeability in the microwave regime of the electromagnetic spectrum is necessary. For the measurement of $\varepsilon, \mu$ several techniques have been proposed [4]. The majority of these concern the estimation of the electrical permittivity. Only a few concern the magnetic permeability. In a paramagnetic sample the magnetic permeability is considered as a scalar quantity and it can be estimated with the Nicolson Rosss method [4]. The permeability of a magnetized ferromagnetic/ferrimagnetic sample, in the microwave regime becomes an asymmetric tensor. The reason for this behavior is resonance precession of the permanent magnetic moment due to the microwave rffield. The aim of this article is to present a new methodology for the estimation of the electrical permittivity and the parameters of the permeability tensor, using rectangular magnetized samples loaded inside a rectangular waveguide.

\section{Theory}

The electromagnetic behavior of a filled waveguide with a magnetized ferrite is generally very complex [6, 7]. A magnetically polarized material with a static magnetic 
field, $\mathbf{H}_{0}=H_{0} \hat{\mathbf{e}}_{y}$ can be described by a non-symmetrical permeability tensor $[1,2]$

$$
[\mu]=\left(\begin{array}{ccc}
\mu & 0 & -j \kappa \\
0 & \mu_{0} & 0 \\
j \kappa & 0 & \mu
\end{array}\right)
$$

where, $\mu=\mu^{\prime}-j \mu^{\prime \prime}, \kappa=\kappa^{\prime}-j \kappa^{\prime \prime}$ with

$$
\begin{gathered}
\mu^{\prime}=\mu_{0}\left(1+\left[\omega_{0} \omega_{m}\left(\omega_{0}^{2}-\omega^{2}\right)+\omega_{0} \omega_{m} \omega^{2} \alpha^{2}\right] / \Delta\right) \\
\mu^{\prime \prime}=\mu_{0} \alpha \omega \omega_{m}\left[\omega_{0}^{2}+\omega^{2}\left(1+\alpha^{2}\right)\right] / \Delta \\
\kappa^{\prime}=\mu_{0}\left[\omega \omega_{m}\left(\omega_{0}^{2}-\omega^{2}\left(1+\alpha^{2}\right)\right] / \Delta\right. \\
\kappa^{\prime \prime}=\mu_{0} 2 \omega_{0} \omega_{m} \omega^{2} \alpha / \Delta
\end{gathered}
$$

$\Delta=\left[\omega_{0}^{2}-\omega^{2}\left(1+\alpha^{2}\right)\right]^{2}+4 \omega_{0}^{2} \omega^{2} \alpha^{2}, \quad \omega_{0}=\gamma \mu_{0} H_{0} \quad$ the Larmor precession frequency, $\omega_{m}=\gamma \mu_{0} M_{s}, \quad M_{s}$ the saturation magnetization, $\gamma$ is the gyromagnetic ratio of the electron, $\mu_{0}$ is the permeability of the free space, and $\alpha$ a phenomenological parameter describing the losses.

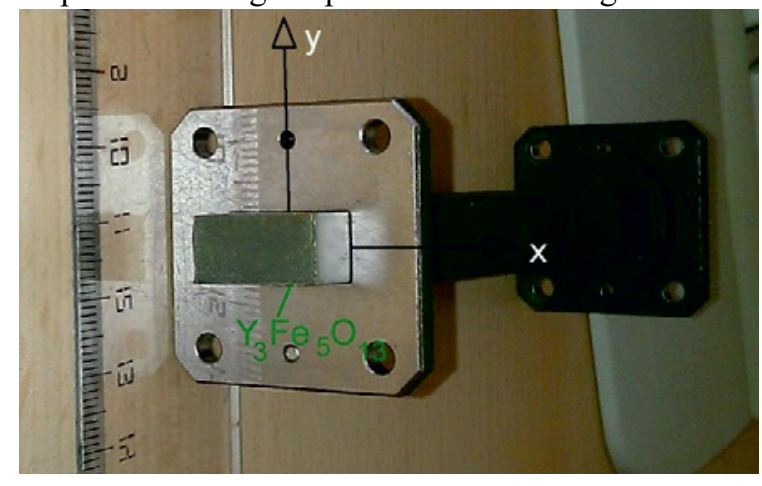

Fig.2 Rectangular wave guide used in the measurements.

In an magnetized sample with infinity dimensions, depending on the propagation configuration, one can define three types of effective magnetic permeability: 1) propagation parallel to the external magnetic field, $\mu_{ \pm}=\mu_{0}(\mu \pm \kappa)$, for right and left circularly polarized waves. 2) Propagation perpendicular to the applied dc magnetic field $\left(\boldsymbol{\beta} \perp \mathbf{H}_{0}\right)$, and rf-electric field parallel to the $\mathbf{H}_{0}, \mu=\mu_{0}$. 3) Propagation perpendicular to the applied dc magnetic field $\left(\boldsymbol{\beta} \perp \mathbf{H}_{0}\right)$, and rf-electric field perpendicular to the $\mathbf{H}_{\mathbf{0}}$,

$$
\mu_{e}=\left(\mu^{2}-\kappa^{2}\right) / \mu
$$

In a rectangular wave guide partially loaded with ferrite magnetically polarized along the $y$-axis in $\mathrm{TE}_{10}$ approximation the propagation constant $\beta$ is given by the roots of the transcendental equation [8]:

$$
\begin{aligned}
& \tan K_{2} a_{f}\left[\mu_{e}^{2} K_{1}^{2}-\mu_{0}^{2}\left\{K_{2}^{2}+\left(\frac{\beta \kappa}{\mu}\right)^{2}\right\} \tan K_{1} x_{0 f} \tan \left[K_{1}\left(a-x_{0 f} a_{f}\right)\right]\right. \\
& +\mu_{0} \mu_{e} K_{1} K_{2}\left[\tan K_{1} x_{0 f}+\tan K_{1}\left(a-x_{0 f}-a_{f}\right)\right] \\
& -\mu_{0} \mu_{e} K_{1} \frac{\beta \kappa}{\mu} \tan K_{2} a_{f}\left[\tan K_{1} x_{0 f}-\tan K_{1}\left(a-x_{0 f}-a_{f}\right)\right]=0,
\end{aligned}
$$

where, $K_{1}^{2}=\omega^{2} \varepsilon_{0} \mu_{0}-\beta^{2}$, and $K_{2}^{2}=\omega^{2} \varepsilon \mu_{e}-\beta^{2}$.
When the waveguide is completely filled with ferrite ( $a_{f}=a$ ) then the propagation constant is given by

$$
\beta=\frac{2 \pi}{\lambda}=\sqrt{\omega^{2} \varepsilon \mu_{e}-\left(\frac{\pi}{a}\right)^{2}} .
$$

If the losses are ignored the effective permeability $\mu_{e}$ can be positive, zero or negative. It is obvious that for negative $\mu_{e}$ propagation does not exist.

\section{Results}

The samples were prepared with standard solid state reaction. Stoichiometric amounts of raw materials $\mathrm{Y}_{2} \mathrm{O}_{3}$, $\mathrm{Fe}_{2} \mathrm{O}_{3}, \mathrm{NiO}$ and $\mathrm{ZnO}$ are thoroughly mixed and heated at $1425^{\circ} \mathrm{C}$ for the $\mathrm{Y}_{3} \mathrm{Fe}_{5} \mathrm{O}_{12}$ and at $1200^{\circ} \mathrm{C}$, for $\mathrm{NiFe}_{2} \mathrm{O}_{4}$ and $\mathrm{ZnFe}_{2} \mathrm{O}_{4}$ with intermediate grindings. In the final step powders are pressed in rectangular bars of appropriate dimensions to adjust the rectangular waveguide and sintered for $24 \mathrm{~h}$. Rietveld refinements of the $\mathrm{x}$-ray diffraction patterns have shown that all the samples are single phase materials with good crystallinity. The x-ray diffraction pattern of $\mathrm{Y}_{3} \mathrm{Fe}_{5} \mathrm{O}_{12}$ can be refined with garnet crystal structure. Similarly, $\mathrm{NiFe}_{2} \mathrm{O}_{4}$ has been successfully refined with the so-called reverse spinel structure. All the $\mathrm{Ni}^{2+}$ ions occupy the octahedral sites while the Fe ions are distributed both on octahedral and tetrahedral sites. On the other hand, the X-ray diffraction pattern of $\mathrm{ZnFe}_{2} \mathrm{O}_{4}$ can be refined with the normal spinel structure. The magnetic properties of the samples are estimated using a SQUID magnetometer.

Fig. 1 shows the magnetization curves of the $\mathrm{Y}_{3} \mathrm{Fe}_{5} \mathrm{O}_{12}$, $\mathrm{NiFe}_{2} \mathrm{O}_{4}$ and $\mathrm{ZnFe}_{2} \mathrm{O}_{4}$. All the samples are soft ferrimagnets. The coercive fields for the $\mathrm{Y}_{3} \mathrm{Fe}_{5} \mathrm{O}_{12}$ and $\mathrm{NiFe}_{2} \mathrm{O}_{4}$ spinel are less than $10 \mathrm{Oe}$, while for $\mathrm{ZnFe}_{2} \mathrm{O}_{4}$ approximately 100 Oe. Interestingly, the saturation magnetization is about $4 \pi M_{s}=3300 \mathrm{G}$ for $\mathrm{NiFe}_{2} \mathrm{O}_{4}$, $4 \pi M_{s} \approx 1800 \mathrm{G}$ for $\mathrm{Y}_{3} \mathrm{Fe}_{5} \mathrm{O}_{12}$ and only $4 \pi M_{s} \approx 120 \mathrm{G}$ for $\mathrm{ZnFe}_{2} \mathrm{O}_{4}$. Obviously these values influence the frequency variation of the effective permeability. Theoretical simulations are performed using the microwave studio of CST package [5]. The ferrite samples are inserted at the center of a rectangular precision wave guide $3 / 4 \times 3 / 8 \mathrm{in}^{2}$ with length $128 \mathrm{~mm}$ (see Fig 2). The scattering parameters $S_{i j}$ are measured using a vector network analyser (Anritzu 37269B).

The standard TRL calibration procedure is used to the zero reference planes. The external magnetic field is applied using two rectangular $\mathrm{Nd}_{2} \mathrm{Fe}_{14} \mathrm{~B}$ permanent magnets with energy product $100 \mathrm{MJ}$. The magnetic circuit is closed with a steel yoke.

Fig. 3 shows the frequency variation of the real part of the effective permeability (Eq. 1.2) for the three studied samples, in several dc magnetic fields, using the values for $M_{\text {s }}$ estimated from magnetization measurements. The Lande factor is taken $g=2$ while the loss parameter $\alpha=\mu_{0} \gamma \Delta H / 2 \omega_{1}$ is selected to have appreciable coincidence among experimental and simulated scattering parameters (see below). Fig. 4 shows the frequency 
variation of the experimental amplitude of the scattering parameters $\left|S_{11}\right|$ and $\left|S_{21}\right|$, measured in several dcmagnetic fields for the $\mathrm{Y}_{3} \mathrm{Fe}_{5} \mathrm{O}_{12}$. The magnetic field is applied parallel to the short dimension of the wave guide (y-axis).

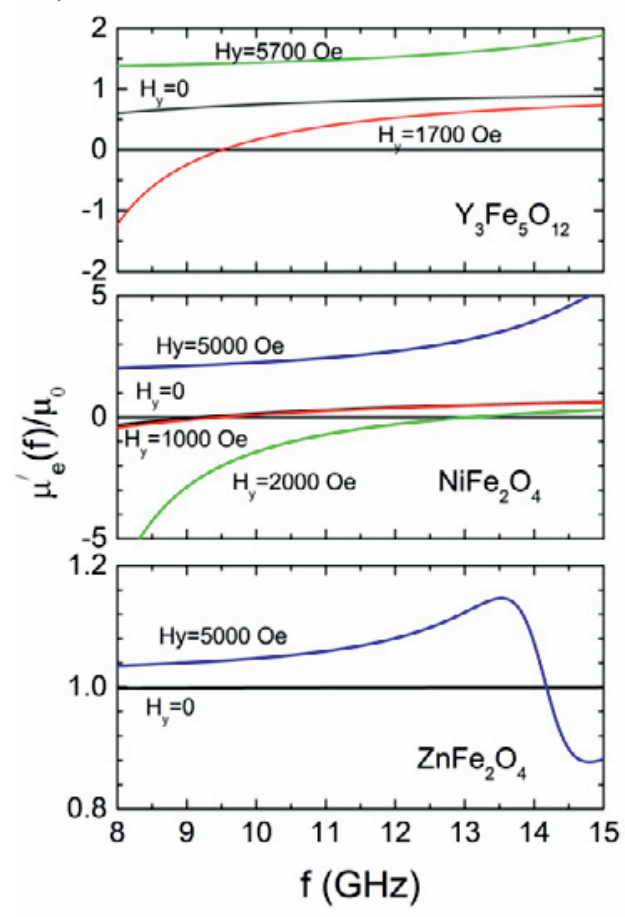

Fig. 3. Theoretical curves for the frequency variation of the $\mu_{e}^{\prime} / \mu_{0}$ (Eq. 1.2) for $\mathrm{Y}_{3} \mathrm{Fe}_{5} \mathrm{O}_{12}, \mathrm{NiFe}_{2} \mathrm{O}_{4}$, and $\mathrm{ZnFe}_{2} \mathrm{O}_{4}$.

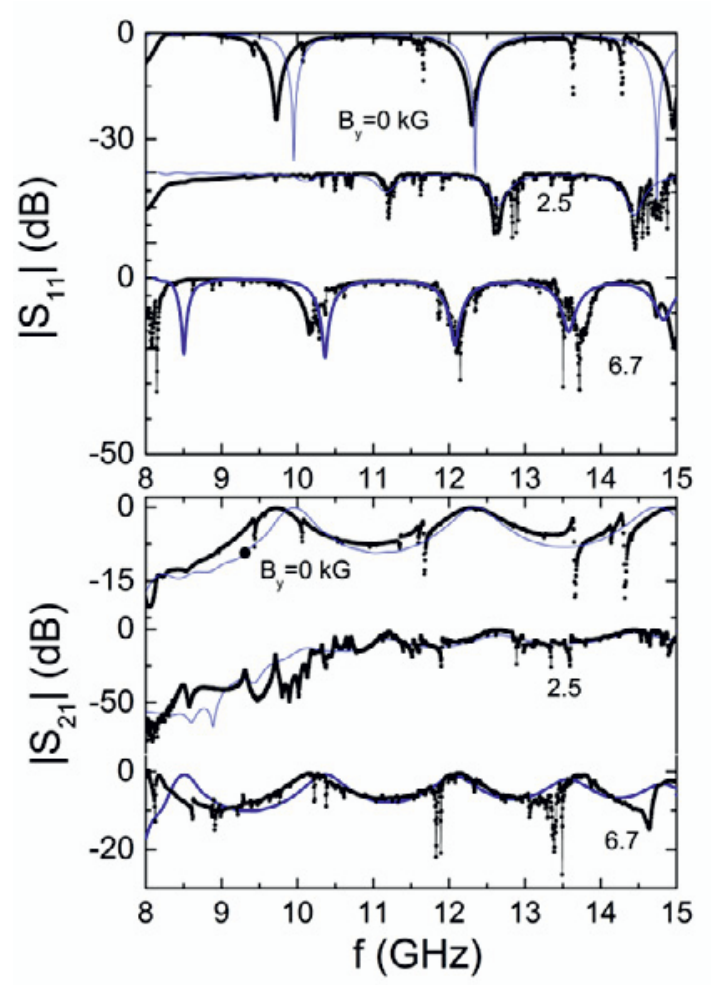

Fig. 4. Amplitude of the scattering coefficients $\left|S_{11}\right|$ and $\left|S_{21}\right|$ versus frequency of $\mathrm{Y}_{3} \mathrm{Fe}_{5} \mathrm{O}_{12}$, for $H_{y}=0,2.5$ and $6.7 \mathrm{KOe}$. The blue solid lines represent theoretical simulations. The sample's dimensions are $9.32 \times 18.8 \times 15.1 \mathrm{~mm}^{3}$.
The continuous lines represent theoretical simulations of the problem using the CST microwave studio solver. In the studied frequency range for $B_{y}=0,\left|S_{11}\right|$ displays three negative peaks $\sim-25 \mathrm{~dB}$. The corresponding scattering parameter $\left|S_{21}\right|$, at the same frequencies, take values near zero. The particular behaviour is related with the reflectionless condition occurring when the thickness of the sample is integer multiplayer of the half wave length of the field inside the material $(d=n \lambda / 2)$. In addition, narrow negative peaks about $\sim-10 \mathrm{~dB}$ are also present. These peaks most probably arise from partially filling of the wave guide cross section with the ferrite or from magnetostatic modes. When the width of the dielectric is less than the wave guide's width the body resonances are shifted in respect to the ones occurring for perfectly loaded case
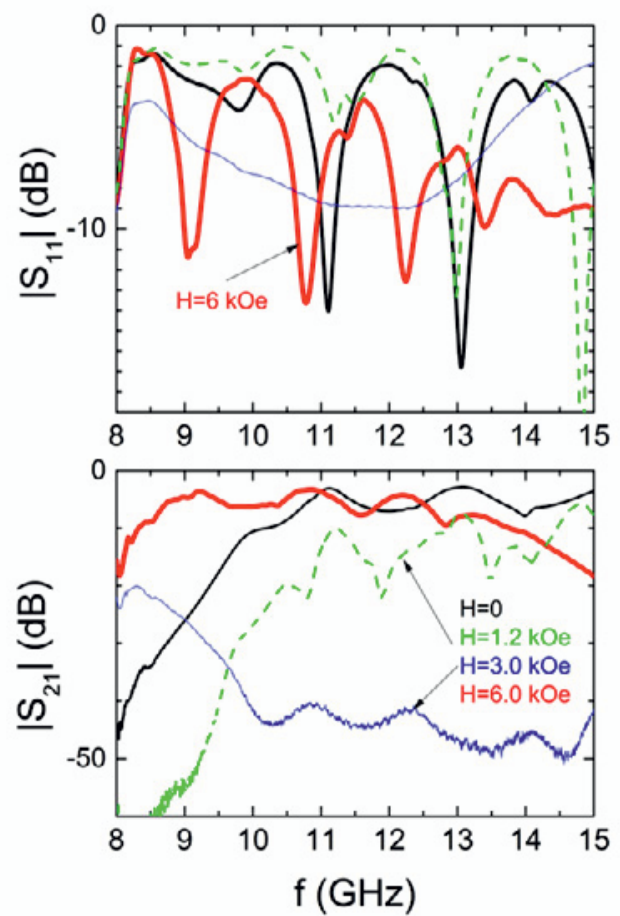

Fig. 5. Amplitude of the scattering coefficients $\left|S_{11}\right|$ and $\left|S_{21}\right|$ versus frequency for $\mathrm{NiFe}_{2} \mathrm{O}_{4}$ in several magnetic fields $(\mathbf{H} \| y-$ axis). The sample's dimensions are $9.2 \times 19.4 \times 18.8 \mathrm{~mm}^{3}$.

This arises from the fact that the dispersion relation $\beta(\omega)$ is shifted. In the other case where $b_{f}<b$ except the shifting of the body resonances, new modes are predicted [8]. For nominal magnetic field $H_{\mathrm{y}}=2.5 \mathrm{kOe}$, a large part of $\left|S_{11}(f)\right|$ has a value near zero and the corresponding $\left|S_{21}(f)\right|$ takes values less than $-40 \mathrm{~dB}$. This behaviour originates from the negative values of the effective permeability $\mu_{e}$ (see Fig. 3). Above a certain frequency $\left|S_{11}(f)\right|$ displays the reflectionless negative peaks a fact which implies $\mu_{e}>0$. For higher nominal fields the frequency range, where $\mu_{e}<0$ is located on the right side, outside the studied frequency range. Clearly, the measurements with nominal field $H_{y}=6.7 \mathrm{kOe}$, the frequency range where $\mu_{e}<0$ is above $15 \mathrm{GHz}$. In order 
to estimate the electric permittivity and the parameters of $\mu_{e}$ we carry out theoretical simulations using the microwave studio of the CST package. Representative examples of the theoretical curves are shown in Fig. 4 with solid lines. Good agreement among theoretical and experimental curves, for zero field has been achieved when the relative electric permittivity for the $\mathrm{Y}_{3} \mathrm{Fe}_{5} \mathrm{O}_{12}$ is $\varepsilon_{r}^{\prime}=16.5$ and $\tan \delta=0.001$. It should be noted that the matching of the reflectionless modes permit a very accurate estimation of $\varepsilon_{r}^{\prime}$. For non-zero magnetic fields, in addition with $\varepsilon_{r}^{\prime}=16.5$ it is necessary one to invoke a saturation magnetization $4 \pi M=1800 \mathrm{G}$ (in agreement with magnetization measurements) a $\Delta H=100$ Oe and a demagnetization correction of the external magnetic field.
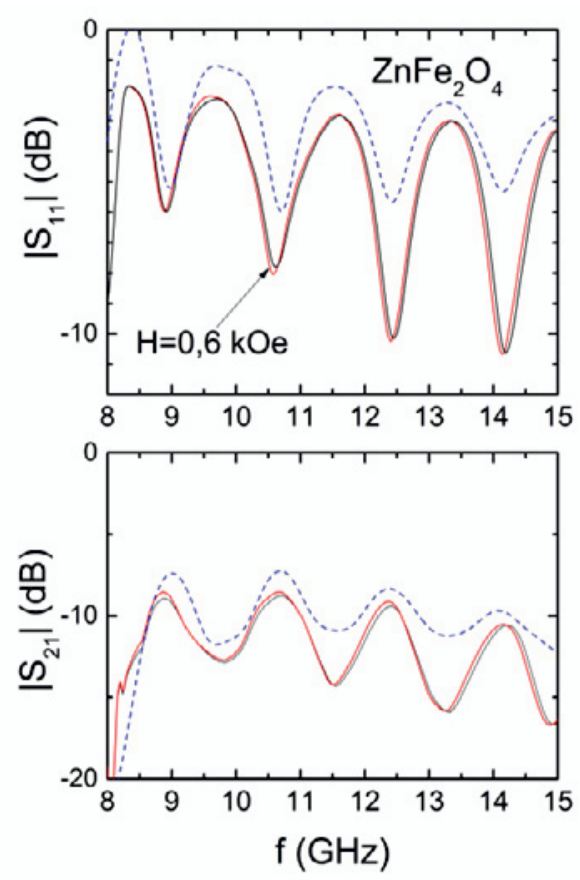

Fig. 6. Scattering parameters $\left|S_{11}\right|$ and $\left|S_{21}\right|$ versus frequency for $H_{\mathrm{y}}=0$ and $6 \mathrm{kOe}$ of $\mathrm{ZnFe}_{2} \mathrm{O}_{4}$. The sample's dimensions are $8.05 \times 17.67 \times 21.33 \mathrm{~mm}^{3}$. The dashed line represents the theoretical calculated values of $\left|S_{11}\right|$ and $\left|S_{21}\right|$ with $\varepsilon_{r}=13.3$ and $\tau=8 \times 10^{-13} \mathrm{~s}$.

Fig. 5 shows the variation with frequency for the amplitude of the scattering parameters $\left|S_{11}\right|$, and $\left|S_{21}\right|$ for $\mathrm{NiFe}_{2} \mathrm{O}_{4}$ spinel measured for $H_{y}=0,1.2,3$ and $6 \mathrm{kOe}$. Simulations of the zero field data demand that for this material the relative permittivity must be $\varepsilon_{r}^{\prime}=12.7$ with, $\tan \delta=0.002$. Based on simulation results in order the experimental results to be reproduced we must take into account the saturation magnetization $4 \pi M_{s}=3300$ Gauss and a losses field width, $\Delta H \approx 300$ Oe. Finally, Fig. 6 shows the frequency dependence for $\left|S_{11}\right|$, and $\left|S_{21}\right|$ scattering parameters, measured for $H_{y}=0$ and $6 \mathrm{kOe}$ for $\mathrm{ZnFe}_{2} \mathrm{O}_{4}$ spinel. In order the reflectionless peaks to be reproduced the permittivity must be $\varepsilon_{r}^{\prime} \approx 13.3$. The $\left|S_{21}\right|$ data reveal Debye type relaxation behaviour with, $\varepsilon_{\infty}=1$ and a relaxation time, $\tau=8 \times 10^{-13} \mathrm{~s}$. The fact that not significant changes are observed between the measurements in zero and $6 \mathrm{kOe}$ magnetic field is directly related with the small frequency variation of the effective permeability for the particular sample (see Fig. 2c). For this sample the variation of $\mu_{e}$ displays the expected resonance behaviour but due to the small saturation magnetization the deviation from the vacuum permeability is small.

\section{Conclusions}

The electric permittivity and the parameters of the magnetic permeability tensor are estimated for the ferrimagnetic samples $\mathrm{Y}_{3} \mathrm{Fe}_{5} \mathrm{O}_{12}, \mathrm{ZnFe}_{2} \mathrm{O}_{4}$ and $\mathrm{NiFe}_{2} \mathrm{O}_{4}$ with parallelepiped shape and using a vector network analyzer equipped with a calibrated rectangular wave guide. The electric permittivity can be estimated from the body resonances $(d=n \lambda / 2)$ if a sufficient long sample is used. The estimation of the magnetic permeability tensors' parameters can be estimated comparing the experimental results with computer simulations using the magnetic properties of the materials from the magnetic measurements.

Acknowledgements: This work is part of the "MAGELLAN" research project, funded by "THALIS" Research Programme of Greek Ministry of Education, Lifelong Learning and Religious Affairs in the framework of NSRF 2007-2013, co-financed by Greece and the European Union.

\section{References}

1. R. E. Collin Field Theory of Guided Waves 2th edition, IEEE Press.

2. David Pozar, Microwave Engineering, Fourth Edition, chapter 9.

3. T. Zervos, A. Alexandridis, F. Lazarakis, M. Pissas, D. Stamopoulos, E. Angelopoulos, K. Dangakis, IET Microwaves Antennas \& Propagation, 6, 158 (2012).

4. A. M. Nicolson and G. F. Ross, IEEE Transaction on Instrumentation and Measurements IM-19, 377 (1970); L. F. Chen, C. K. Ong, C. P. Neo, V. V. Varadan and V. K. Varadan, " Microwave Electronics, Measurements and materials Characterization, John Wiley \& Sons, Ltd, (2004).

5. CST Microwave studio, Computer simulation technology AG, Darmstadt, Germany, 2012.

6. G. Barzilai and G. Gerosa, IRE Transactions on Antennas and Propagation, AP-7, S471 (1959); G. Barzilai and G. Gerosa, II Nuovo Cimento, X-7,685, (1958).

7. P. Queffelec, M. Le Floc'h and P. Gelin, IEEE Transactions on Microwave Theory and Techniques 48, 1344, (2000); P. Queffelec, M. medium Le Floc'h and P. Gelin, IEEE Transactions on Microwave Theory and Techniques, 47, 390 (1999) and references therein.

8. M. L. Kales, H. N. Chait and N. G. Sakiotis, J. Appl. Phys. 24, 816 (1953). 